This item was submitted to Loughborough's Institutional Repository by the author and is made available under the following Creative Commons Licence conditions.

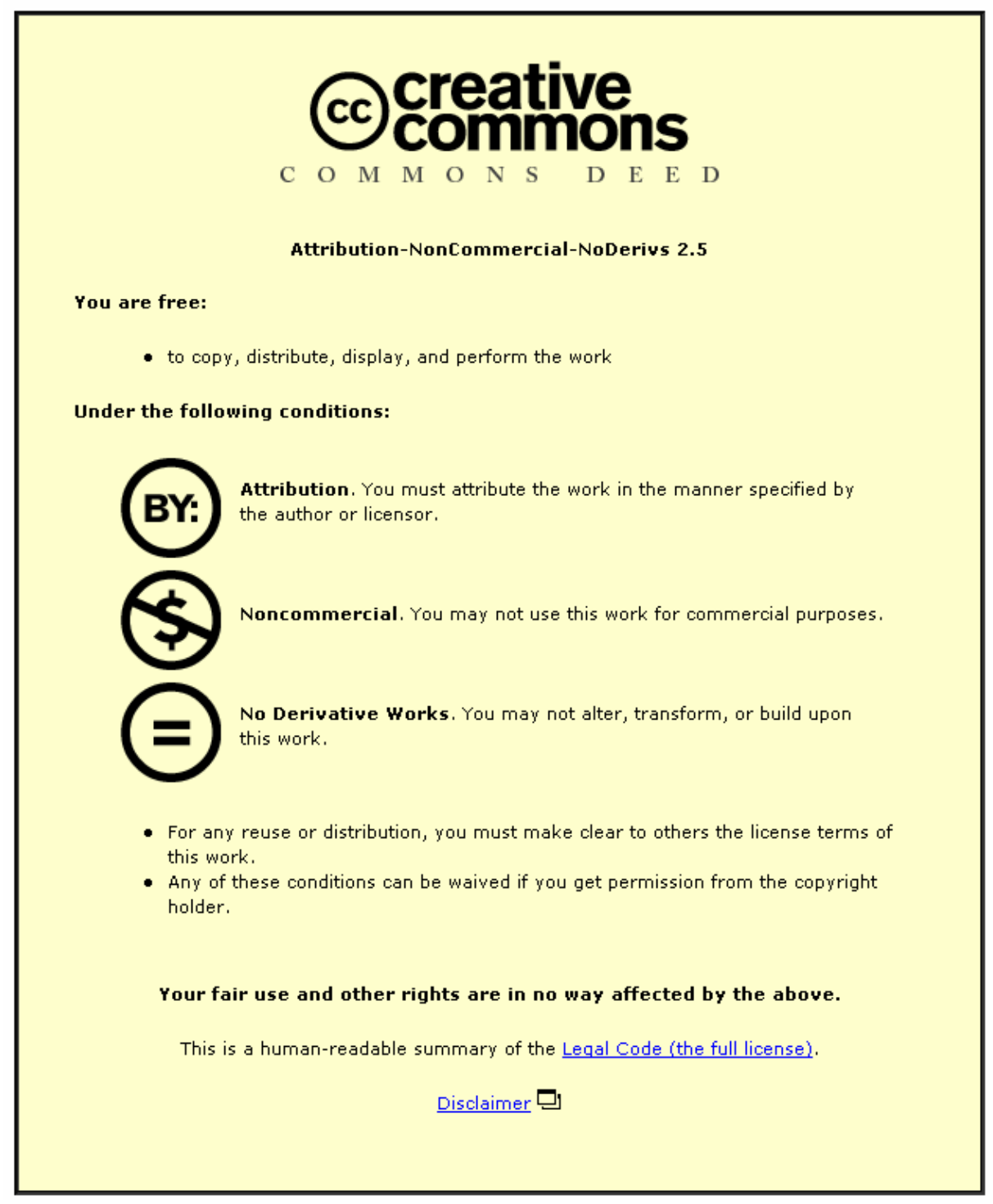

For the full text of this licence, please go to: http://creativecommons.org/licenses/by-nc-nd/2.5/ 


\title{
Application of the Digraph Method in System Fault Diagnostics
}

\author{
E. M. Kelly \& L. M. Bartlett \\ Aeronautical and Automotive Engineering Department, Loughborough University, \\ Loughborough, Leicestershire, LE11 3TU, UK. T:+44(0)1509 227276, F:+44(0)1509 227275. \\ E.M.Kelly@lboro.ac.uk/L.M.Bartlett@lboro.ac.uk
}

\begin{abstract}
There is an increasing demand for highly reliable systems in the safety conscious climate of today's world. When a fault does occur there are two desirable outcomes. Firstly, detection is required to discover whether functionality at a pre-determined level can be maintained and secondly, a necessary repair strategy is sought to minimise system disruption.

Traditional focus on fault diagnosis has been through a sequential testing procedure or via real time mechanisms. The limitations have incorporated the issue of combining real time diagnosis; enabling fast analysis, with multiple fault causes. These issues are typical of critical situations within current complex system architectures.

The diagnostic method suggested in this paper uses the digraph procedure, which represents the propagation of inputs through a system. The procedure involves generating a model; linking nodes referring to system parameters and determining the relationship(s) which connect the nodes. Fault detection occurs by means of tracing through the diagram. The method has been applied to a water tank system during steady state operation. Diagnosis is conducted by comparing readings from sensors in the system with expected readings, given the system mode of operation. The results demonstrate the effective use of this technique for fault diagnosis of the application system.

Keywords: Digraphs, Availability, Failure Detection, Fault Diagnosis.
\end{abstract}

\section{Introduction}

Fault diagnosis has become a highly important facet of engineering applications. Information is required about possible failures which may disrupt the running of a system and thus decrease its effectiveness. The requirements for systems which are safety critical are based on high availability along with mechanisms to reduce downtime. It is imperative that these attributes are maintained with continual enhanced system complexity involving integrated computer and mechanical systems.

Fault diagnosis is concerned with identifying and isolating the cause of a system malfunction. Detection and isolation can occur either continuously, and thus detect faults in real-time, or at a specific point in time. Ideally fault diagnosis should also deal with multiple faults since it is common for complex engineering applications to have more than one fault causing a system malfunction. Worthy of note is that multiple failures could be caused through interdependencies between components.

More recent approaches have considered using reliability assessment tools, such as failure modes and effects analysis (FMEA) and fault tree analysis (FTA), as opposed to conducting diagnostics through testing techniques. Price[1,2] demonstrated that the process of FMEA could be automated and thus used within a diagnostic tool employing qualitative model-based reasoning as a means of diagnosing multiple faults within a system. A method has also been produced which will predict system diagnosability during the early design phase through using information obtained from both FMEA and FTA[3]. The limiting factor involved with this process is that it is not possible to generate all combinations of failures for large systems due to the vast calculation time required.

A current method by Hurdle et al. considers system fault diagnostics through using FTA[4]. In this case fault trees, using both coherent and non-coherent methods, were generated for stated top events. Coherent fault trees use both AND and OR logic and only consider component failure events, whilst noncoherent fault trees also use NOT logic, which allows for the inclusion of both failed and working component events. Hurdle et al. developed five schemes, of differing complexity, which could be used to model system behavioural deviations using sensor information and thus perform fault diagnostics. The most successful scheme involved the use of non-coherent fault trees with additional specific consistency checks. The 
suitability of the method is unknown should the number of sensors used for fault diagnosis and also the size of the system be increased.

This paper describes a method which utilises digraphs to model fault propagation through a simple system. It is then possible to perform fault diagnostics based upon the data contained within the system digraph. Digraph models have been incorporated into, and used with other reliability tools such as fault trees and HAZOP analysis[5-8], to analyse various system performance measures.

In terms of using digraphs for fault diagnosis, Rao[9] addresses the problem of diagnosing single faults in systems. The single fault diagnosis problem primarily deals with identifying the set of all likely fault sources which correspond to a given set of outcomes. Iverson and Pattersine-Hine[10] extend this approach to also consider the combination of two failures via an AND gate. This method has a use in real-time automated monitoring and diagnosis, although its limitation lies in the number of potential fault causes. A variant stemming from Digraphs, called Signed Directed Graphs (SDG)[11] have also been used for fault diagnosis in process plants[12].

The aim of the method proposed in this paper is to cope with multiple fault possibilities and also enable real time diagnosis. More than one fault often causes identical or similar symptoms in current systems. Diagnosis therefore depends on isolating the primary causal faults from a series of observable effects i.e. sensor deviations. Section 2 considers the modelling of fault propagation in systems through using digraphs. Section 3 incorporates a description of a simple water tank system which contains two integrated control loop structures. A system digraph of the simple system is formed in Section 4 where fault diagnostic analysis considers the system in steady state. Section 5 provides the conclusions of the research.

\section{The digraph method}

A digraph, also known as a directed graph, illustrates the fault propagation through a system. It comprises a set of nodes and edges; where the nodes (V) represent the system process variables and edges (E), the relationships between the nodes. The general digraph model is represented by: $\mathrm{DG}=(\mathrm{V}, \mathrm{E})$

The edges (lines) connecting the nodes within a digraph represent the interrelations between process variables within an engineering application. Examples of process variables include temperature, mass flow, pressure and signals from sensors. The standard notation for representing process variable deviations within the digraph are expressed as one of five discrete values: $+10,+1,0,-1,-10$, representing respectively; large high, moderate high, normal, moderate low and high low. A simple digraph is shown in Figure 1.

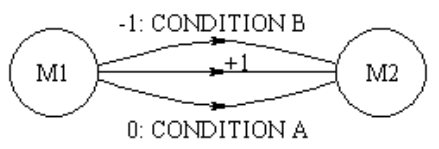

Figure 1 A simple digraph representation

From Figure 1 it can be seen that M1 and M2, the nodes, are connected by three edges. The alphanumeric code M1 represents mass flow at location one and M2 the mass flow at location two. M1 is the independent variable whilst M2 is the dependent variable since a directed edge connects M2 to M1. The edge with a gain of +1 is a normal edge since this represents the relationship which is usually true. The second and third edges are conditional edges since their relationship is only true whenever the condition represented by ':' exists. It must be noted that only one edge is true at any one time. Any gain associated with an edge is equal to $\delta Y / \delta X$. In the case of Figure 1:

$$
\text { Gain }=\delta(\mathrm{M} 2) / \delta(\mathrm{M} 1)
$$

M1 and M2 thus display a positive relationship under normal conditions.

The digraph method has an ability to clearly depict control loops. This is illustrated in more detail in Section 4.2. A control loop comprises three main elements; a sensor, controller and control device. Two basic forms of control loops which can be represented by digraphs are:

1. Negative Feedback Loops (NFBL)

Any moderate disturbances within process variables are corrected by this loop. Its path on a digraph starts and ends on the same node and the product of all of the normal gains around the NFBL are negative.

2. Negative Feedforward Loops (NFFL)

In theory any disturbances within process variables can be cancelled by this loop. However, in practise exact cancellation does not take place. It is represented as two or more paths from one node to another node on a digraph and the sign of the product of all of the normal gains is different on each of the paths.

Note: The same discrete values which are used to represent the gains associated with an edge within a digraph are also employed to describe the effect a disturbance has on a particular process deviation.

An unexpected process deviation(s) within a system is represented by 'highlighting' the respective node in the digraph. Subsequent propagation of the deviation 
through the system is represented by marking all of the nodes which were affected by the initial highlighting. A simple example is shown in Figure 2. A large positive disturbance noted at node M3, represented as M3(+10), results in the commencing of back-tracing from this point. The faults which could have taken place giving rise to $\mathrm{M} 3(+10)$ are:

$\mathrm{M} 3(+10) \rightarrow$ Fault Three, $\mathrm{M} 3(+10) \rightarrow \mathrm{M} 2(+10) \rightarrow \mathrm{M} 1(+10) \rightarrow$ Fault One.

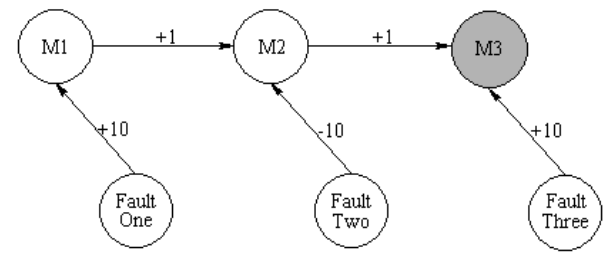

Figure 2 Digraph back-tracing example

\section{The Water Tank System}

The water tank system constitutes a simple system which is used as a means of investigating the suitability of the digraph method in the field of fault diagnostics.

The tank system is illustrated in Figure 3. The aim of the system is to maintain the water level between pre-determined levels, monitored by level sensor one, $\mathrm{S} 1$. Under normal conditions water would flow in through Valve One (V1) and out through Valve Two (V2). Should the water level rise above a set point then the level control system would close Valve One and not allow any more mains water into the tank. Valve Three (V3) acts as the safety valve for the system. Should the tank overflow, or leak through either a rupture or hole, the overspill tray located underneath would collect the 'lost' water. A more comprehensive description of the water tank system is given in Section 3.1.

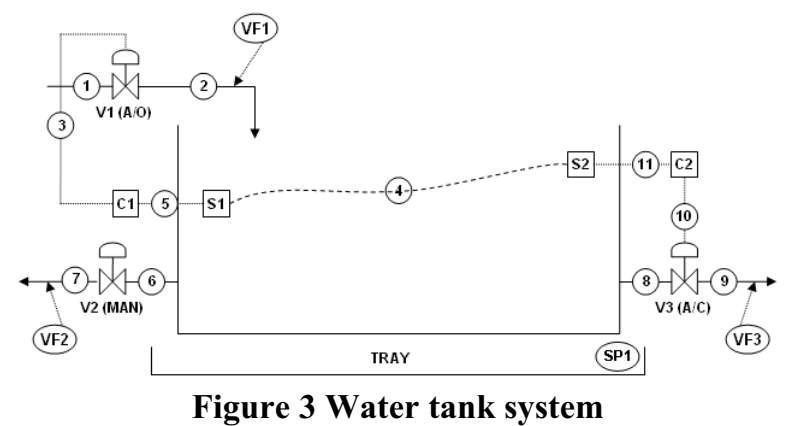

\subsection{System description}

The water tank system in Figure 3 consists of three valves; V1-3, two level sensors; S1/2, and their respective control units; $\mathrm{C} 1 / 2$, plus an overspill tray;
TRAY. There are also six labelled sections of pipe; P12, P6-7 and P8-9.

Valve One is an air-to-open $(\mathrm{A} / \mathrm{O})$ inlet valve controlled by $\mathrm{C} 1$. S1 monitors the level of the water within the tank and relays information to $\mathrm{C} 1$; should the water level, under normal conditions, fall below a predetermined lower limit then the controller unit (C1) will open V1, thus allowing mains water into the tank. Conversely, should the water level rise above an upper limit monitored by $\mathrm{S} 1$ then the valve is closed by the controller. Valve Two is simply a manual (MAN) outlet valve which is controlled by an operator and therefore has no control loops directly associated with it. Valve Three is an air-to-close (A/C) outlet valve controlled by $\mathrm{C} 2$. V3 is part of the safety loop and therefore under normal operating conditions will not come into use. However; should a failure occur and the water level rise above a pre-determined upper limit (monitored by S2) C2 would open V3 as a means of reducing the water level, so preventing a water tank overflow situation. Should the water tank overflow then the overspill tray would collect the water. Control Loop Two is therefore redundant until there is a failure within Control Loop One.

\subsection{Sensor locations}

The water tank system status can be obtained through using information from three flow sensors; VF1-3 used for measuring flow from valves V1-3 respectively. The flow sensors are able to detect either a presence or absence of flow of water. A fourth sensor, referred to as SP1, is located in the overspill tray, therefore indicating whether any water has leaked from the tank. These sensors are known as system observation points and it is assumed that for the purposes of this research the sensors are sufficient and reliable.

\subsection{Operating modes}

The water tank system has two operating modes. Namely, 'ACTIVE' when the operator opens V2, or 'DORMANT', when V2 is closed. During the 'ACTIVE' mode water is removed from the tank via V2 and refilled by the mains supply through V1. There should be no water flow through V3 or any indication of water in the overspill tray. In the 'DORMANT' mode the system is simply in standby mode with no flow registering through any of the valves or water in the overspill tray. Table 1 shows the sensor readings which would be expected during the two operating modes. 
Table 1 System operating mode sensor scenarios

\begin{tabular}{ccccc}
\hline Operating Mode & VF1 & VF2 & VF3 & SP1 \\
\hline ACTIVE & Flow & Flow & No Flow & No Water \\
DORMANT & No Flow & No Flow & No Flow & No Water \\
\hline
\end{tabular}

\subsection{Water tank system scenarios}

There are sixteen possible scenarios in which the water tank system could be in when considering all potential sensor readings from VF1-3 and SP1. The scenarios (Scen) are outlined in Table 2, where; $\mathrm{F}=$ flow, $\mathrm{NF}=$ no flow, $\mathrm{W}=$ water and $\mathrm{NW}=$ no water.

Table 2 Water tank system scenarios

\begin{tabular}{|c|c|c|c|c|c|c|c|c|c|}
\hline Scen & VF1 & VF2 & VF3 & SP1 & Scen & VF1 & VF2 & VF3 & SP1 \\
\hline 1 & F & F & F & W & 9 & F & $\mathrm{NF}$ & F & W \\
\hline 2 & F & F & F & NW & 0 & F & $\mathrm{NF}$ & F & $\mathrm{NW}$ \\
\hline 3 & $\mathrm{NF}$ & F & F & W & 11 & F & F & $\mathrm{NF}$ & W \\
\hline 4 & $\mathrm{NF}$ & F & F & NW & 12 & F & F & $\mathrm{NF}$ & $\mathrm{NW}$ \\
\hline 5 & $\mathrm{NF}$ & $\mathrm{NF}$ & F & W & 13 & $\mathrm{NF}$ & F & $\mathrm{NF}$ & W \\
\hline 6 & $\mathrm{NF}$ & $\mathrm{NF}$ & $\mathrm{F}$ & NW & 14 & $\mathrm{NF}$ & $\mathrm{F}$ & $\mathrm{NF}$ & NW \\
\hline 7 & $\mathrm{NF}$ & NF & $\mathrm{NF}$ & W & 15 & $\mathrm{~F}$ & $\mathrm{NF}$ & $\mathrm{NF}$ & W \\
\hline 8 & $\mathrm{NF}$ & $\mathrm{NF}$ & $\mathrm{NF}$ & NW & 16 & F & $\mathrm{NF}$ & $\mathrm{NF}$ & $\mathrm{NW}$ \\
\hline
\end{tabular}

From Table 2 it can be seen that the 'ACTIVE' and 'DORMANT' operating modes of Table 1 correspond to scenarios 12 and 8 respectively. All other scenarios represent a deviation in the expected behaviour and hence indicate the presence of potential faults in the system.

\subsection{Component failure modes}

Table 3 contains all possible component failure modes considered in this research, which could affect the functionality of the water tank system of Figure 2.

Table 3 Component Failure modes

\begin{tabular}{|c|c|c|c|}
\hline Code & Component Failure & Code & Component Failure \\
\hline $\begin{array}{l}\mathrm{P}_{\mathrm{B}} \mathrm{B}(1-2,6-7, \\
8-9)\end{array}$ & Pipe $P_{\mathrm{i}}$ is blocked & $\begin{array}{l}\mathrm{S}_{\mathrm{i}} \mathrm{FS} \\
(1 \leq \mathrm{i} \geq 2)\end{array}$ & Sensor $\mathrm{S}_{\mathrm{i}}$ fails stuck \\
\hline $\begin{array}{l}\mathrm{P}_{\mathrm{i}} \mathrm{R}(1-2,6-7, \\
8-9)\end{array}$ & Pipe $P_{i}$ is ruptured & $\begin{array}{l}\mathrm{C}_{\mathrm{C} F H} \\
(1 \leq \mathrm{i} \geq 2)\end{array}$ & Controller $\mathrm{C}_{\mathrm{i}}$ fails high \\
\hline $\begin{array}{l}\mathrm{V}_{\mathrm{iFC}} \\
(1 \leq \mathrm{i} \geq 3)\end{array}$ & Valve $V_{i}$ fails closed & $\begin{array}{l}\mathrm{C}_{\mathrm{C} F L} \\
(1 \leq \mathrm{i} \geq 2)\end{array}$ & Controller $\mathrm{C}_{\mathrm{i}}$ fails low \\
\hline $\begin{array}{l}\mathrm{V}_{\mathrm{F}} \mathrm{i} \\
(1 \leq \mathrm{i} \geq 3)\end{array}$ & Valve $V_{i}$ fails open & $\begin{array}{l}\mathrm{C}_{\mathrm{FS}} \\
(1 \leq \mathrm{i} \geq 2)\end{array}$ & Controller $\mathrm{C}_{\mathrm{i}}$ fails stuck \\
\hline $\begin{array}{l}\mathrm{V}_{\mathrm{i}} \mathrm{FS} \\
(1 \leq \mathrm{i} \geq 3)\end{array}$ & Valve $V_{i}$ fails stuck & NMWS & No mains water supply \\
\hline $\begin{array}{l}\mathrm{S}_{\mathrm{F}} \mathrm{FH} \\
(1 \leq \mathrm{i} \geq 2)\end{array}$ & Sensor $S_{i}$ fails high & TL & Water tank leaks \\
\hline $\begin{array}{l}\mathrm{S}_{\mathrm{i}} \mathrm{FL} \\
(1 \leq \mathrm{i} \geq 2)\end{array}$ & Sensor $S_{i}$ fails low & TR & Water tank ruptured \\
\hline WOST & Water in over-spill tray & & \\
\hline
\end{tabular}

\section{Application of digraph methodology}

\subsection{Procedure}

The procedure identified for developing digraphs is: a) Define system to be analysed.

b) List all component failures of the system.

c) Separate the system into sub-units and components.

d) Identify and classify control loops, if present.

e) Generate the digraph models for the sub-units taking into consideration all process variable deviations which could have an effect on the variables in the model. Also consider the extent of the effect the process variable deviations may have on the system with regards to the assigning of discrete values to the deviations.

f) Form system digraph model by connecting common variables from the sub-unit models.

g) Diagnosability is conducted through a process of back-tracing (discussed in Section 4.3) from known deviating nodes within the system digraph.

The end result of the system digraph model for the water tank system is illustrated by Figure 7 . The intermediary stages, which were undertaken before the final system digraph model was generated, are shown in Figures 4-6. The process of fault diagnostics which occurs once the system digraph has been developed is discussed in detail in Section 4.3.

\subsection{Development of the water tank system digraph}

The assumptions made during the construction of the water tank system unit digraph models were:

a) Given a pipe rupture no flow is registered by the flow sensors.

b) There is greater volume loss through a tank rupture compared with a tank leakage.

c) System is in steady state condition.

The unit digraph models of Figures 4-6 are derived for all three valves. They consider the way in which the components function with regards to the basic physics laws governing mass, energy and momentum. The alphanumeric label within the nodes either represents the process variable or component failure mode. The numeric section of the label corresponds to a specific location in the water tank system illustrated by Figure 3. The precursor to the numeric section represents one of the following process variables:

- ' $\mathrm{M}$ ' : mass flow

- 'L' : level

- $\quad$ ' $\mathrm{P}$ ' : pressure (force)

The process flow and control loop structure of the system is illustrated by Figures 4-6. The nodes representing the component failure modes can either cause disturbances to the process variables or nullify a relationship between process variables, as shown by the 
arcs connecting the nodes. Further failure modes are illustrated through the use of conditional arcs between process variables.

Control loops within the water tank system are represented as negative feedback loops (NFBL), where the product of the normal gains around the loop is negative. NFBL's are used since they introduce the ability to correct any moderate disturbances which may be present in one of the process variables. When tracing faults through a NFBL, it must be considered in its entirety. Moderate and large disturbances must be considered as well as inactive control devices or those failing either high or low, so resulting in a disturbance entering the loop. If this was not adhered to, then in the case of Figure 4, back tracing round the loop from a large negative disturbance at L4, L4(-10), would result in $\mathrm{L} 4(-10) \rightarrow \mathrm{M} 2(-10) \rightarrow \mathrm{P} 3(-10) \rightarrow \mathrm{P} 5(+10) \rightarrow$ L4 $(+10)$. This exemplifies the reasoning behind not simply back tracing round the loop since the scenario produces inconsistent results[5].

Figure 4 illustrates the combined unit digraph of V1 and its respective control loop. The air-to-open control valve, V1, is represented by the relationship between $\mathrm{M} 1, \mathrm{M} 2$ and P3. The control loop associated with V1 is illustrated by the negative feedback loop of M2-L4-P5P3; where the product of the normal gains around the loop is negative. Due to the inverse acting relationship between the sensor and control unit (P5-P3) the normal arc is 'signed' as negative. Since V1 is an air-to-open valve the normal arc between P3 and M2 is 'signed' as positive.

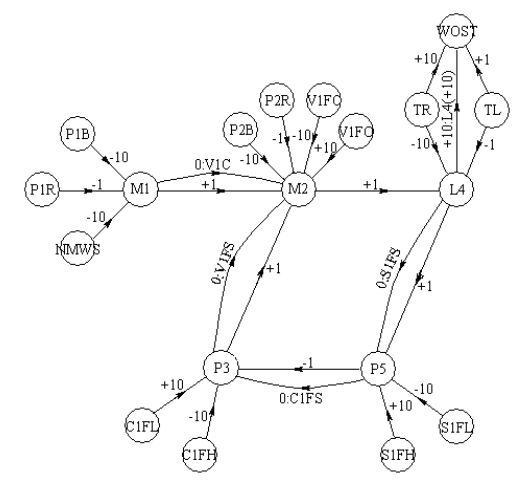

Figure 4 Valve one and control loop one unit digraph

Mass flow, M1, entering V1 could be affected by three possible component failure modes; P1B, P1R and NMWS. Each of these failure modes could cause a reduction in mass flow at location one. The mass flow exiting V1, represented by M2, could be affected either by a disturbance within the control loop M2-L4-P5-P3 or one which has previously occurred at M1. There are also four failure modes directly associated with M2.
These are P2B, P2R, V1FC and V1FO. V1FO, unlike $\mathrm{P} 2 \mathrm{~B}, \mathrm{P} 2 \mathrm{R}$ and $\mathrm{V} 1 \mathrm{FC}$ could cause a positive deviation in mass flow at location two. If $\mathrm{V} 1$ is simply closed in the 'DORMANT' mode then the relationship between M1 and M2 is nullified, as shown by the conditional arc joining the two nodes.

Nodes P5 and P3 are part of the NFBL incorporating M2 and L4. The pressure of the output signal from level sensor $\mathrm{S} 1$ is positively dependent on the level of water in the tank. The relationship between the level sensor output node, P5 and the tank level node, L4 is nullified if the level sensor is stuck. Node P5 is positively affected by the failure mode S1FH and negatively influenced by S1FL. Node P3 represents the output from controller unit $\mathrm{C} 1$ to $\mathrm{V} 1$. The inverse relationship between P5 and P3 is nullified if $\mathrm{C} 1$ is stuck. In a similar manner to P5 there are two failure modes associated with P3. P3 is positively affected by $\mathrm{C} 1 \mathrm{FL}$ and negatively by $\mathrm{C} 1 \mathrm{FH}$. The mass flow exiting $\mathrm{V} 1$, represented by node M2 is positively dependent on $\mathrm{P} 3$. This positive relationship is nullified if $\mathrm{V} 1$ fails stuck.

The tank level node, L4, is directly affected by the failure modes TR and TL. Both TR and TL can lead to the situation of water in the overspill tray, represented by node WOST. The tank level is positively dependent on the mass flow entering through V1.

Since V2 is a manually operated valve there are no control loops associated with the unit digraph model as illustrated by Figure 5. The relationships between the nodes have been established in a similar manner to V1.

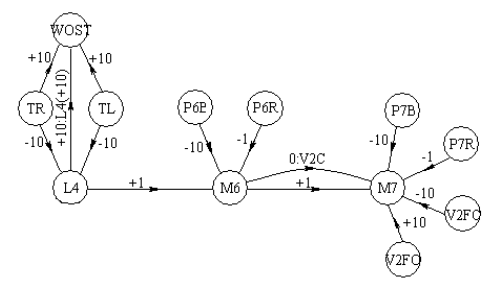

Figure 5 Valve two unit digraph

Figure 6 illustrates the combined unit digraph of V3 and its respective control loop. The air-to-close control valve, V3, is represented by the relationship between M8, M9 and P10. The control loop is illustrated by the negative feedback loop of L4-P11-P10-M8. Again, due to the inverse acting relationship between P11 and P10, the normal arc is 'signed' as negative. The normal arc between P10 and M8 is also 'signed' as negative due to $\mathrm{V} 3$ being an air-to-close valve. 


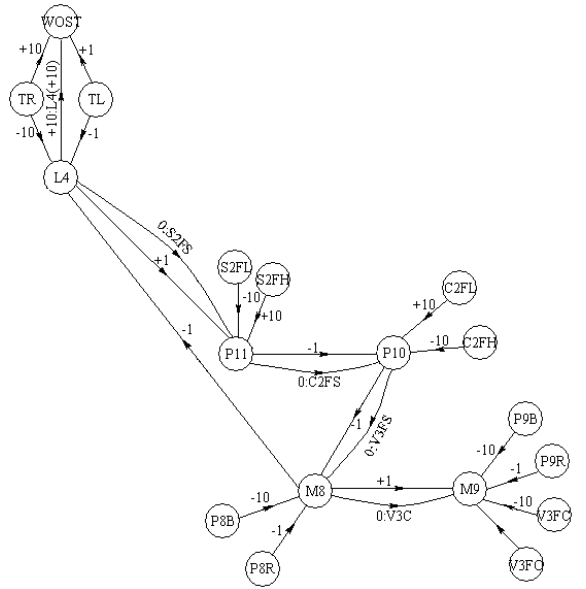

Figure 6 Valve three and control loop two unit digraph

The complete water tank system digraph illustrated in Figure 7 is formed by combining the unit digraphs of Figures 4-6.

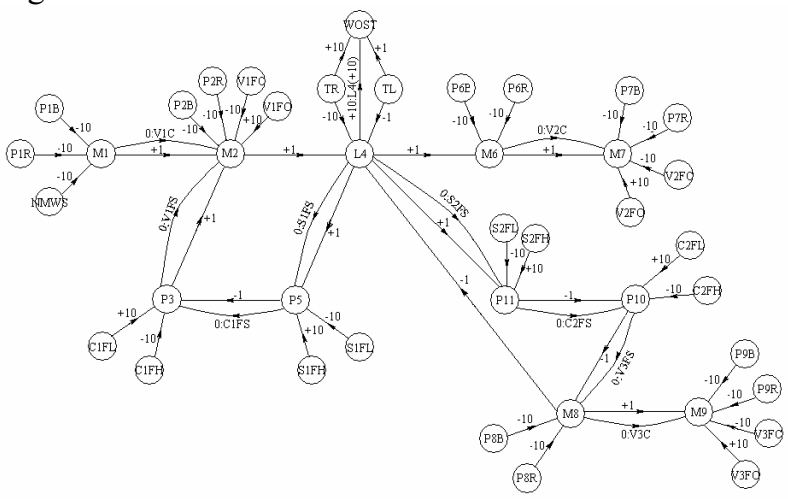

Figure 7 Water tank system digraph

\subsection{Digraph diagnostic methods}

The use of digraphs in fault diagnosis is based on comparing system sensor readings with those which are expected whilst the system is in a known operating mode. If a sensor registers a deviation, diagnosis involves back-tracing through the system digraph from the node which represents the location of the given deviation. Through back-tracing it is possible to determine which failure modes may have attributed to the process variable deviation.

When the water tank system is in the 'ACTIVE' state the expected sensor readings are as outlined in Table 4. The system can deviate from the 'ACTIVE' mode through one or more of its sensor readings, thus changing from the known operating mode to one of the possible scenarios previously outlined in Table 2 . With regards to the 16 scenarios listed some noted a single deviating sensor, whilst others noted multiple deviations. A deviating sensor indicates that a fault has occurred within the system. The results achieved from using the digraph method to determine which faults could have led to a deviation registered by the sensors in the water tank system are discussed in Section 4.4. A similar process could be conducted if it were assumed that the water tank system is in the 'DORMANT' mode.

Table 4 ACTIVE mode sensor readings

\begin{tabular}{ccccc}
\hline $\begin{array}{c}\text { Operating } \\
\text { Mode }\end{array}$ & VF1 & VF2 & VF3 & SP1 \\
\hline ACTIVE & Flow & Flow & No Flow & No Water \\
\hline
\end{tabular}

Two methods were considered for assessing the application of the water tank system digraph to the deviated scenarios. The methods differ in their approach to back-tracing through the digraph. Backtracing refers to the manner in which an analyst moves from a deviating node through the digraph until all possible failure modes, which could have contributed to the deviation, are noted. The logic employed by each method is:

1) Method One: This approach requires the user to fully back-trace through the digraph model from the node registering a deviation until a point is reached where no further back-tracing can be conducted.

The application of method one revealed that many fault options, some contradictory, are produced for a given deviating sensor reading. This can be attributed to the amount of back-tracing conducted.

Due to the deficiencies of results produced from using method one, another method was required to remove conflicting results for a particular system deviation; this in turn led to the derivation of method two.

2) Method Two: In this approach information from sensor readings is used to flag nodes (and in some cases whole digraph sections) representing process variables which are known to be not deviating. Back-tracing from a known deviating node ceases once the analyst reaches a flagged section. Method two is further described in Section 4.4.

For multiple deviating sensors, method two is applied in the same way, with the results for each deviation being ANDed together.

\subsection{Application of digraph method two to a scenario}

One scenario, 'FS16' (Table 5) has been chosen to demonstrate the diagnostic capability of the water tank system digraph. 'FS16' encompasses sensor readings which have been obtained whilst the system is 
supposed to be in the 'ACTIVE' state. 'FS16' deviates from the 'ACTIVE' state through sensor VF2 registering the situation 'no flow'. The remaining three sensor readings of VF1, VF3 and SP1 do not differ from the expected 'ACTIVE' state readings.

Table 5 'FS16' deviating sensor readings

\begin{tabular}{ccccc}
\hline $\begin{array}{c}\text { Operating } \\
\text { Mode }\end{array}$ & VF1 & VF2 & VF3 & SP1 \\
\hline ACTIVE & Flow & Flow & No Flow & No Water \\
FS16 & Flow & No Flow & No Flow & No Water \\
\hline
\end{tabular}

The diagnostic results obtained from method two are thus expanded. A brief discussion of the results is conducted in Section 4.4.1.

Node M7 is addressed since it represents the status of mass flow leading away from Valve 2. M7 is therefore 'marked' on the digraph. The procedure followed is:

1) From Figure 7 the fault propagation causing a large negative disturbance $\mathrm{M} 7(-10)$ is determined; a large negative disturbance is considered since this takes into account the 'no flow' registered by VF2.

2) From 'FS16' it is noted that only VF2 registers a deviation from the ACTIVE mode. Consequently three sections of the digraph corresponding to the non-deviating sensors VF1, VF3 and SP1 are flagged: sections incorporating control loop one (L4-P5-P3M2), control loop two (L4-P11-P10-M8) and water in the overspill tray (WOST). Backtracing from the deviating node of M7 ceases at node L4 (reference Figure 7).

3) From reviewing the system digraph, it is evident that the faults capable of causing a direct negative disturbance of node $\mathrm{M} 7$ are: V2FC, P7R and P7B. Following the fault propagation to node $\mathrm{M} 6$, the process deviations P6R and P6B could be a further cause for $M 7(-10)$. No further faults are documented due to the flagging of the other digraph sections, back-tracing therefore ceases at L4(-10).

All of the diagnostic results obtained in (2) are a possible cause for the given deviation registered at VF2. The results are also consistent with the state of the water tank sensors. There are no additional causes since it is assumed that there are no further faults with the system due to the remaining three sensors not registering any deviation from the ACTIVE state.

4.4.1 Digraph method two conclusions. Diagnostic results for the sixteen scenarios arise from partial backtracing through the water tank system digraph. It is assumed non-deviating sensor readings indicate the absence of any possible faults. When comparing methods one and two, method two produces less fault combinations for a given deviation due to the flagging of sections.

There is however one concern with the flagging method when dealing with the safety system in the ACTIVE mode. Given the scenario that there is a failure of Control Loop One, Control Loop Two is required. If however there is a failure within this loop, the sensor reading registers the expected state, i.e. no flow at V3, when in fact there is a fault. This only occurs for one scenario when there is an overflow situation. To overcome this problem a condition is prewritten into the digraph to prevent the 'flagging' response. The flagging of non-deviating sections during back-tracing acts as a form of consistency check in method two. This removes conflicting results which display erroneous inconsistencies between sensor readings.

\section{Conclusion}

Through using digraphs there is the ability to back trace within the model thus locating failures associated with a given event. A system digraph presents a clear representation of the relationships between output and input variables. It is also possible to 'hone-in' on sections within the model so creating the capacity to ignore all other interrelationships, if required. This introduces the ability to visualise how much one specific failure may affect a variable.

Digraphs are suited to illustrating fault propagation through a system as they closely reflect the physical structure of the system under investigation. Fault propagation is depicted through direct and indirect influences of a faulty component. The development of a system diagraph requires a thorough understanding of the system and individual components. This process is aided by the high standard of current engineering drawings.

In using the approach both methods assume nondeviating sensor readings indicate the absence of a fault(s) and therefore a simple sensor check would involve switching the operating mode of the tank system. For example, in 'FS16' only VF2 deviates from the ACTIVE mode so switching the tank system to the DORMANT mode would yield any errors, if present, with VF1, VF3 and SP1. This is especially relevant to those components not displaying any deviation from the normal operating mode of the system.

Future work in applying the methodology to a larger system of increased complexity would illustrate the effects of multiple faults more effectively. The work described in the preceding sections does not take into account transient effects, the dynamics of the 
system are an additional feature to be investigated. Ongoing research proposes the application of the digraph method to a simulation test stand of an aircraft fuel system. This aids in furthering the applicability of the method described to other systems which better represent larger engineering systems. The inclusion of dynamic effects would advance the real-time diagnosis of the fuel rig.

From the research which has been conducted it can be stated that digraphs have been proven to provide valid results when considering the fault diagnostics of a water tank system with control features in steady state.

\section{References}

[1] PRICE, C. and TAYLOR, N., 1997, Multiple Fault Diagnosis from FMEA, Proceedings from the National Conference on Artificial Intelligence, pp1052.

[2] PRICE, C., 1997. AutoSteve: Electrical Design Analysis. Colloquium Digest - IEE, 338(4).

[3] HENNING, S. and PAASCH, R., Diagnostic Analysis for Mechanical Systems, Proceedings of the ASME Design Engineering Technical Conferences, 2000 pp391.

[4] HURDLE, E.E., BARTLETT, L.M. and ANDREWS, J.D., System Fault Diagnostics Using Fault Tree Analysis, Proceedings of the 16th Advances in Reliability Technology Symposium, 2005.

[5] ANDREWS, J.D. and MORGAN, J.M., 1986. Application of the Digraph Method of Fault Tree Construction to Process Plant. Reliability Engineering, 14(2), pp. 85.

[6] KOHDA, T. and HENLEY, E.J., 1988. On Digraphs, Fault Trees and Cut Sets. Reliability Engineering and System Safety, 20(1), pp. 35.

[7] ANDREWS, J. and BRENNAN, G., 1990. Application of the Digraph Method of Fault Tree Construction to a Complex Control Configuration. Reliability Engineering and System Safety, 28(3), pp. 357.

[8] VAIDHYANATHAN, R. and VENKATASUBRAMANIAN, V., 1995. Digraph-based models for Automated HAZOP Analysis. Reliability Engineering and System Safety, 50(1), pp. 33.

[9] RAO, N. S. V., 1993. Expected-Value Analysis of Two Single Fault Diagnosis Algorithms. IEEE Transactions on Computers, 42(3), pp. 272.

[10] IVERSON, D.L. and PATTERSINE-HINE, F.A., 1995. Advances in Digraph Model Processing Applied to Automated Monitoring and Diagnosis. Reliability Engineering and System Safety, 49(3), pp. 325.
[11] VEDAM, H. and VENKATASUBRAMANIAN, V., 1997. Signed Digraph Based Multiple Fault Diagnosis. Computers Chemical Engineering (supp), 21, pp. S655.

[12] PALMER, C. and CHUNG, P.W.H., 2000. Creating Signed Directed Graph Models for Process Plants. Industrial and Engineering Chemistry Research, 39(7), pp. 2548. 